\title{
Vortex Methods for the Simulation of Turbulent Flows: Review*
}

\author{
Rio YOKOTA** and Shinnosuke OBI*** \\ ** Department of Mechanical Engineering, Boston University, \\ 110 Cummington St., Boston, MA, 02215, U.S.A. \\ *** Department of Mechanical Engineering, Keio University, \\ 3-14-1 Hiyoshi, Kouhoku-ku, Yokohama 223-8522, Japan \\ E-mail: obsn@mech.keio.ac.jp
}

\begin{abstract}
Vortex methods are a group of Lagrangian and semi-Lagrangian methods based on the vorticity-streamfunction or vorticity-velocity formulation of the Navier-Stokes equation, and provide an interesting alternative to grid based methods for external flows dominated by unsteady vortical motion. In the present review article, we will assess the advantages and disadvantages of vortex methods for the simulation of incompressible turbulent flows, based on the speed and accuracy benchmarks that have been performed recently. Our goal is to objectively and quantitatively evaluate the performance of this non-standard method, by directly comparing it's speed and accuracy against finite difference and pseudo-spectral DNS codes under identical calculation conditions. We also present examples of vortex methods in engineering applications of turbulent flows.
\end{abstract}

Key words : Vortex Methods, Particle Methods, Isotropic Turbulence, Homogeneous Shear Flow, Turbulent Channel Flow, Fast Multipole Methods, MDGRAPE, GPU

\section{Introduction}

Vortex methods are a group of Lagrangian and semi-Lagrangian methods based on the vorticity-streamfunction or vorticity-velocity formulation of the Navier-Stokes equation. Vortex methods possess the following advantages over grid based methods. First of all, vortex methods calculate the convection in a Lagrangian manner by moving the calculation points according to the local velocity. Thus, it is free of numerical diffusion and dispersion, and allows the use of larger time step sizes because it is not subject to numerical instabilities arising from the convection. This feature alone allows the vortex method to become an order of magnitude faster than finite difference methods. ${ }^{(1)}$ Furthermore, the velocity-vorticity formulation allows the vortex method to place calculation points only in the regions of non-zero vorticity. For external flows, this results in a large reduction of the number of calculation points because the vorticity is confined to a thin region near the wall. It is also possible to use adaptive spatial refinement without agonizing over the interconnectivity of calculation points. Furthermore, the high acceleration rate of these methods on graphics processing units (GPUs) ${ }^{(2)}$ could become an advantage in the near future.

It is also true that vortex methods face numerous challenges when solving engineering applications in turbulence. For example, mesh based methods use boundary fitted meshes with high aspect ratio to capture the large velocity gradients in the wall normal direction. In theory, vortex methods can do the same by using anisotropic elements with high aspect ratio near the wall. However, such methods are still under development and have not been successfully implemented in practical vortex method calculations. Another challenge is the relatively high calculation cost of the fast multipole method (FMM), when compared to fast Poisson solvers using multigrid methods or FFT. For the same number of points/particles the FMM is several

*Received 2 Aug., 2010 (No. R-10-0323) [DOI: 10.1299/jfst.6.14]

Copyright $\subset 2011$ by JSME 
orders of magnitude larger than fast Poisson solvers. Though, it is important to keep in mind how the FMM, multigrid method, and FFT each scale differently on massively parallel architectures.

In the present review article, we will assess the advantages and disadvantages of vortex methods for the simulation of incompressible turbulent flows, based on the speed and accuracy benchmarks that have been performed recently. Our goal is to objectively and quantitatively evaluate the performance of this non-standard method, by directly comparing it's speed and accuracy against finite difference and pseudo-spectral DNS codes under identical calculation conditions. The paper is organized as follows. First, a brief history of vortex methods is given in Section 2, which explains the evolution of vortex methods according to the computers and numerical methods that were available at the time. In Section 3, the numerical method is explained with minimum mathematical complexity. In Section 4, both software and hardware acceleration techniques are presented along with results. In Section 5, two validation studies are presented, each with a different purpose. In Section 6, an example of the application of vortex methods to engineering problems are shown. Finally, the conclusions are given in Section 7.

\section{Brief History of Vortex Methods}

The origins of the vortex method can be traced back to the inviscid two dimensional calculations of the Kelvin-Helmholtz instability by Rosenhead. ${ }^{(3)}$ The elements used in this calculation were singular point vortices, which tracked the motion of the vortex sheet rollup. The study of Rosenhead was followed by similar calculations using regularized point vortices, which resulted in a more stable calculation. ${ }^{(4)(5)}$ The convergence of the two dimensional point vortex method for Euler's equations was proven by Hald. ${ }^{(6)}$ Proof was provided for the existence of a solution as long as the vortex blobs overlapped sufficiently. This was extended to three dimensions by Beale \& Majda ${ }^{(7)}$ using Lagrangian update to compute the stretching. Another ancestor of the vortex method is the panel method ${ }^{(8)}$, which is a method that solves the potential flow around a body of arbitrary shape. It has been widely used in aeronautical industries during the 1980's and early-90's. ${ }^{(8)}$ The panel method is nowadays considered as a special case of the boundary element method. ${ }^{(9)}$ Actually, all the techniques mentioned above can be integrated into a single framework, as the solution of the Poisson equation using Green's functions. ${ }^{(10)}$ Therefore, the detailed understanding of the individual methods mentioned above is not a prerequisite for understanding modern vortex methods.

The extension to viscous flows has been the focal point of interest in vortex methods during the 1980's and early 1990's. Among others, the random vortex method (RVM) by Chorin $^{(11)}$, the core spreading method (CSM) by Leonard ${ }^{(12)}$, and the particle strength exchange (PSE) by Degond \& Mas-Gallic, ${ }^{(13)}$ were introduced as methods to calculate the diffusion in vortex methods. The inherent difficulty in calculating the diffusion in a Lagrangian framework has lead to the use of semi-Lagrangian methods such as the vortex-in-cell (VIC). ${ }^{(14)}$ The viscous treatment near the wall has been of prime difficulty and also of prime importance, since the wall diffusion is the source of the entire vorticity in the vortex method simulation. Chorin ${ }^{(15)}$ first proposed a method that used sheet elements that move randomly in the vicinity of a wall. Fishelov ${ }^{(16)}$ used the Prandtl equations for a thin layer to calculate the wall diffusion. Although some of these methods evolved into rigorous direct numerical simulations of the Navier-Stokes equations during the next decade, the methods mentioned above were in no way comparable to finite difference methods of that time, in terms of both accuracy and speed. The intention during this era was not to fully resolve the high Reynolds number fluid flow, but to somewhat mimic the dominant vortex dynamics using discrete vortex elements.

During the 1990's and early 2000's, the viscous vortex method framework was reinforced by the introduction of essential tools. Winckelmans \& Leonard ${ }^{(17)}$ gives a detailed mathematical formulation of the three dimensional viscous vortex method, with particular emphasis on 
the smoothing functions and stretching term calculation. Remeshing techniques to deal with the Lagrangian distortion of vortex elements were also introduced during this period. The use of spatially varying element sizes has been investigated for the $\operatorname{VIC}^{(18)}$ and $\operatorname{PSE}^{(19)}$. Near wall treatment of vortex methods advanced remarkably during this period. Koumoutsakos et al. formulated the basic equations for the two dimensional case. ${ }^{(20)}$ The equations were slightly modified to achieve higher accuracy ${ }^{(21)}$ and extended to three dimensions by Ploumhans et $a l .{ }^{(22)}$ Furthermore, fast algorithms such as the treecode by Barnes \& Hut ${ }^{(23)}$ and the fast multipole method (FMM) by Greengard \& Rokhlin ${ }^{(24)}$ were applied to vortex method calculations. This reduced the calculation cost from $O\left(N^{2}\right)$ to $O(N \log N)$ and $O(N)$ for the treecode and FMM, respectively. Specialized hardware for $N$-body problems were also developed during this period ${ }^{(25)(26)}$. These efforts led to a new paradigm, i.e. solving flows of moderate Reynolds numbers and fully resolving these flows. During this period, vortex methods were applied to numerous complex applications, and their results showed that these methods could indeed produce quantitatively correct results for any kind of flow.

Recent interest in vortex method studies has been focused on the development of more advanced tools, such as fast $\mathrm{N}$-body solvers ${ }^{(27)}$, high order convergent diffusion schemes ${ }^{(28)}$ ${ }^{(29)}$, and efficient three-dimensional near wall treatment ${ }^{(30)}$. Further maturation of each of these tools shall soon allow the vortex method to overcome most of its traditional weaknesses. However, the lack of validation in canonical turbulent flows has left the overall reliability of vortex methods in question. The independent sources of errors must be investigated in simple flows, despite the fact that such flows are not always suitable for showing the advantage of vortex methods. The present contribution provides a summary of a series of validation studies in simple turbulent flows, along with calculation speed benchmarks on next generation hardware.

\section{Vortex Methods}

The present vortex method solves the following set of equations.

$$
\begin{aligned}
& \nabla^{2} \mathbf{u}=-\nabla \times \omega \\
& \frac{D \omega}{D t}=\omega \cdot \nabla \mathbf{u}+v \nabla^{2} \omega
\end{aligned}
$$

where $\mathbf{u}$ is the velocity vector, $\omega$ is the vorticity vector, and $v$ is the kinematic viscosity. When the velocity Poisson equation (1) is formulated as an integral equation using Green's functions, it yields the generalized Biot-Savart equation. In the present vortex method the vorticity equation (2) is solved in a fractional step manner by solving the first and second term on the right hand side, and the left hand side separately.

First, the vorticity field is discretized by using a superposition of Gaussian distributions.

$$
\omega_{i}=\sum_{j=1}^{N} \boldsymbol{\alpha}_{j} \zeta_{\sigma},
$$

where $\alpha$ is the vortex strength, and

$$
\zeta_{\sigma}=\frac{1}{\left(2 \pi \sigma_{j}^{2}\right)^{3 / 2}} \exp \left(-\frac{r_{i j}^{2}}{2 \sigma_{j}^{2}}\right)
$$

is the Gaussian smoothing function. $\sigma$ is often referred to as the core radius, and represents the physical length scale of vortex elements. $r_{i j}$ is the distance between point $i$ and point $j$. In $N$-body calculations, $i$ is referred to as the target, and $j$ is referred to as the source. The generalized Biot-Savart equation can be written as

$$
\mathbf{u}_{i}=\sum_{j=1}^{N} \alpha_{j} g_{\sigma} \times \nabla G
$$


where $G$ is the Green's function for the Laplace equation and

$$
g_{\sigma}=\operatorname{erf}\left(\sqrt{\frac{r_{i j}^{2}}{2 \sigma_{j}^{2}}}\right)-\sqrt{\frac{4}{\pi}} \sqrt{\frac{r_{i j}^{2}}{2 \sigma_{j}^{2}}} \exp \left(-\frac{r_{i j}^{2}}{2 \sigma_{j}^{2}}\right),
$$

is the cutoff function corresponding to the Gaussian distribution in (4).

The left hand side (convection term) of (2) is accounted for by advancing the coordinates according to the local velocity.

$$
\frac{D \mathbf{x}_{i}}{D t}=\mathbf{u}_{i}
$$

The first term on the right hand side (stretching term) of (2)

$$
\frac{D \omega}{D t}=\omega \cdot \nabla \mathbf{u}
$$

is solved by substituting (3) and (5), which yields

$$
\frac{D \boldsymbol{\alpha}_{i}}{D t}=\sum_{j=1}^{n} \boldsymbol{\alpha}_{j} \nabla\left(g_{\sigma} \times \nabla G\right) \cdot \boldsymbol{\alpha}_{i}
$$

In the present calculations, we use the core spreading method (CSM) to solve the second term on the right hand side (diffusion term)

$$
\frac{D \omega}{D t}=v \nabla^{2} \omega
$$

by changing the variance of the Gaussian distribution

$$
\sigma^{2}=2 v t
$$

to account for the diffusion. We also perform radial basis function interpolation for smaller Gaussian distributions $^{(31)}$ to ensure the convergence of the CSM.

In summary, the present vortex method solves equations (5), (7), (9), and (11). Among these, (5) and (9) involve far field interactions and must be solved using fast $N$-body algorithms.

\section{Acceleration of Vortex Methods}

\subsection{Fast Multipole Methods}

There have been numerous attempts to accelerate vortex methods using fast $N$-body algorithms and specialized hardware. Winckelmans et al. ${ }^{(32)}$ used the treecode to accelerate the vortex method calculation and also the boundary element method calculation. Marzouk \& Ghoniem $^{(33)}$ used K-means clustering for load balancing their parallel treecode, and applied the code to the simulation of a transverse jet. Cocle et al. ${ }^{(34)}$ use a hybrid of the Vortex-InCell method and FMM to utilize the benefit of both methods. Sbalzarini et al. ${ }^{(27)}$ developed a particle-mesh library that calculates one vortex method iteration for 268 million particles in 85 seconds using 128 processors. Using this library, Chatelain et al. ${ }^{(35)}$ have performed a calculation of wing tip vortices using 10 billion vortex elements. Sheel et al. ${ }^{(36)}$ used a special purpose computer originally designed for molecular dynamics (MDGRAPE-2) and showed a 100-fold acceleration of the direct interaction calculation. Later, they applied the FMM on the MDGRAPE-3 and observed a 4 times speed-up of the FMM ${ }^{(37)}$.

We will now show a demonstration of the previously mentioned FMM on MDGRAPE-3. The calculation time of the Biot-Savart equation for $N$ particles on the CPU and MDGRAPE3 are shown in Fig. 1. "CPU" refers to the calculations performed on a dual core Xeon $5160(3.0 \mathrm{GHz})$ processor. "MDG3" refers to the calculations on an MDGRAPE-3. The direct method on CPU has a high constant and is of the order $O\left(N^{2}\right)$. The direct method on MDGRAPE-3 has a lower constant but is still proportional to $O\left(N^{2}\right)$. Conversely, the FMM on CPU has a high constant but the order is $O(N)$. The FMM on MDGRAPE-3 has a low constant and is of order $O(N)$. At $N=10^{6}$ the FMM on MDGRAPE-3 is about 16 times faster than the MDGRAPE-3 itself, and approximately 4 times faster than the FMM on the CPU. 


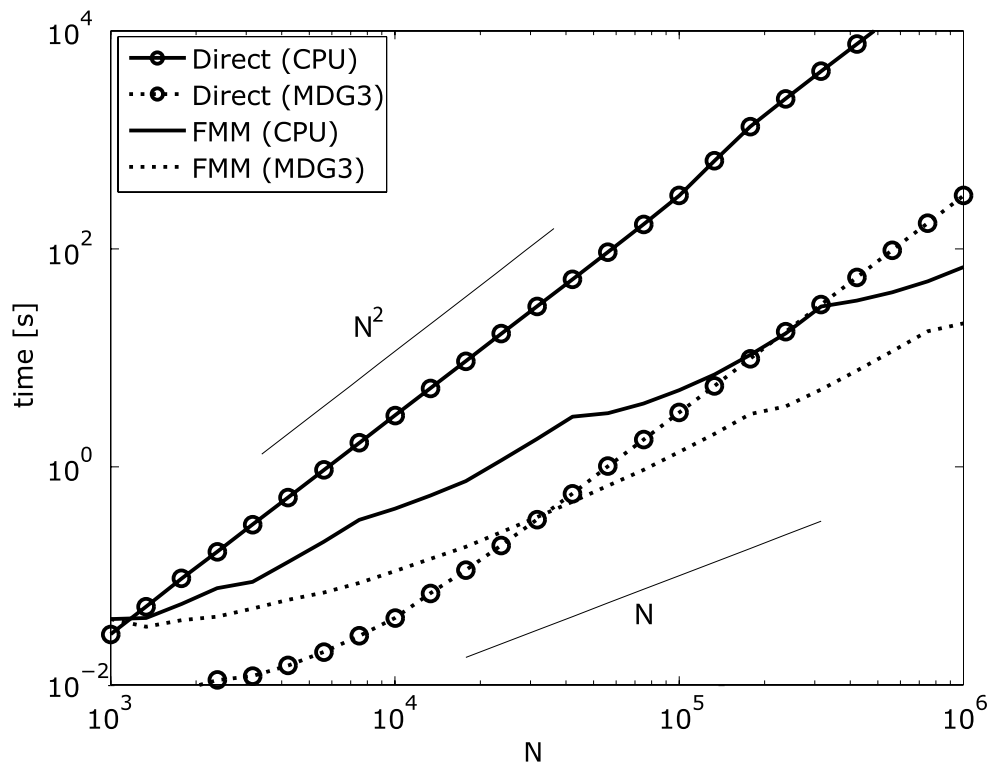

Fig. 1 Calculation time of Biot-Savart equation for $N$ particles on CPU and MDGRAPE- $3^{(37)}$

\subsection{GPU}

A more recent trend in HPC is the development of programmable graphics processing units (GPU). The $N$-body simulations using NVIDIA's CUDA (Compute Unified Device Architecture) programming environment have achieved a performance of over 200 GFlops on a single GPU ${ }^{(38)-(40)}$. Furthermore, Schive et al. ${ }^{(41)}$ used 32 GPUs to accelerate the direct calculation of gravitational interactions, and Stone et al. ${ }^{(42)}$ used 6 GPUs for the calculation of the particle mesh Ewald (PME) method in their MD simulation. Stock \& Gharakhani ${ }^{(43)}$ implemented the treecode on the GPU to accelerate their vortex method calculation. Their treecode was approximately 17 times faster, whereas their direct calculation was 127 times faster on the GPU for $N=500,000$ particles. Similarly, Gumerov \& Duraiswami ${ }^{(44)}$ calcu- $^{2}$ lated the Coulomb interaction using the FMM on GPUs, and achieved a 72-fold speed-up for the FMM, while their direct calculation was 855 times faster on the GPU for $N=1,048,576$ particles. Yokota et al. performed a vortex method calculation of the homogeneous isotropic turbulence on a cluster of 64 GPUs using the $\mathrm{FMM}^{(45)}$. This work was extended to a calculation on 256 GPUs. ${ }^{(2)}$

We now show a demonstration of the FMM on GPUs. The calculation time of the BiotSavart equation for $N$ particles on the CPU and GPU are shown in Fig. 2. "CPU" refers to the calculations performed on an Intel Core i7 $(2.67 \mathrm{GHz})$ processor. "GPU" refers to the calculations on a NVIDIA GeForce GTX 295. All calculations are on a single CPU/GPU. Compared to Fig. 1, the results are quite similar. The direct method accelerates approximately 2 orders of magnitude on the GPU. The FMM accelerates around 30 times. This is much larger than the acceleration rate on the MDGRAPE-3. This is due to the fact that the entire FMM is executed on the GPU, while only part of the FMM can be implemented on the MDGRAPE-3.

\section{Validation of Vortex Methods in Canonical Turbulent Flows}

The simulation of turbulence requires the accurate prediction of the production and dissipation of kinetic energy. In vortex methods, this is made possible by properly calculating the stretching term and diffusion term of the vorticity equation. The homogeneous isotropic turbulence does not involve any mean shear and near wall effects. Therefore, it is suitable for the isolated validation of the viscous diffusion problem, and serves as the starting point of the sequence of validation. The homogeneous shear flow involves mean shear (strong stretching), but no near wall effects. Thus, it serves the role of validating the stretching term, while adding 


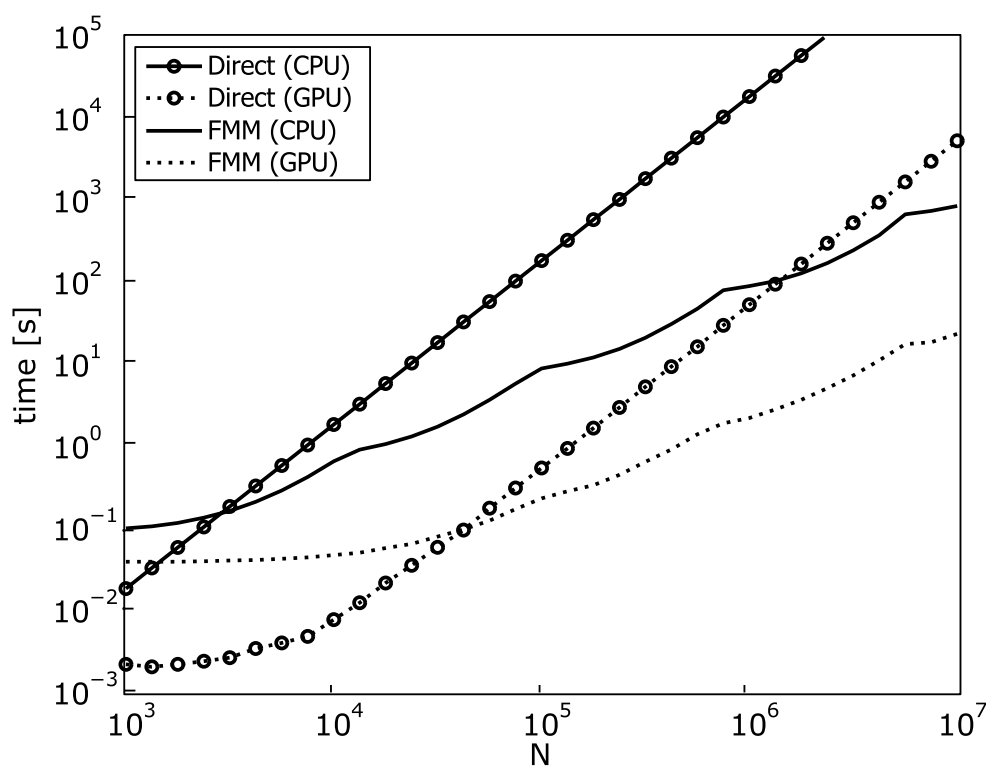

Fig. 2 Calculation time of Biot-Savart equation for $N$ particles on CPU and GPU(45)

minimum complexity to the isotropic case. The turbulent channel flow involves all three difficulties but is still geometrically simple and an equilibrium turbulent flow. The availability of credible references also proved to be helpful when validating the near wall issues of vortex methods.

\subsection{Homogeneous Isotropic Turbulence}

Only a small number of vortex methods have been tested for the homogeneous isotropic turbulence. Cottet et al. ${ }^{(46)}$ used the vortex-in-cell for the viscous diffusion scheme and compared with a pseudo-spectral method for $N=128^{3}$ grid points. Totsuka \& $\mathrm{Obi}^{(47)}$ compared the pseudo-spectral method with the vortex method using the core spreading method and a Laplacian model used in the moving particle semi-implicit (MPS). Yokota et al. ${ }^{(31)}$ compared a pure Lagrangian vortex method against a pseudo-spectral method under identical conditions, and observed a quantitative agreement for the decay rate of kinetic energy and energy spectrum.

The absence of vortex method applications to homogeneous isotropic turbulence can be explained by its comparative inefficiency for this particular flow. The calculation cost becomes high compared to pseudo-spectral methods because vortex methods do not benefit from periodic boundary conditions, whereas the pseudo-spectral methods enormously do. Even with the use of fast algorithms, the speed of a N-body calculation is two orders lower than a grid based fast Poisson solver as noted by Cottet et al. ${ }^{(46)}$. However, with the help of GPUs, the difference between the speed of vortex methods and pseudo-spectral methods would become rather small. ${ }^{(45)}$

The flow field of interest is a decaying isotropic turbulence with an initial Reynolds number of $R e_{\lambda} \approx 100$. The calculation domain is $[-\pi, \pi]^{3}$ and has periodic boundary conditions in all directions. Details of the periodic FMM are shown in our previous publication ${ }^{(31)}$. The number of calculation points was $N=256^{3}=16,777,216$ for both the vortex method and spectral method. The order of multipole expansion was set to $p=10$, and the number of periodic images was $2^{5} \times 2^{5} \times 2^{5}$ for the present calculations. We used a total of 256 GPUs for the calculation of the isotropic turbulence.

The spectral Galerkin method with primitive variable formulation ${ }^{(48)}$ is used in the present study as reference. A pseudo-spectral method was used to compute the convolution sums, and the aliasing error was removed by the $3 / 2$-rule. The time integration was performed using the 


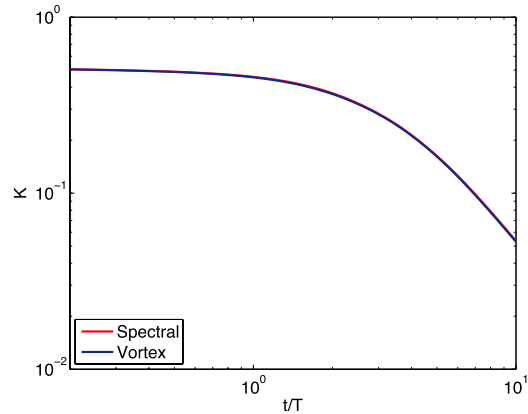

(a) Decay of kinetic energy

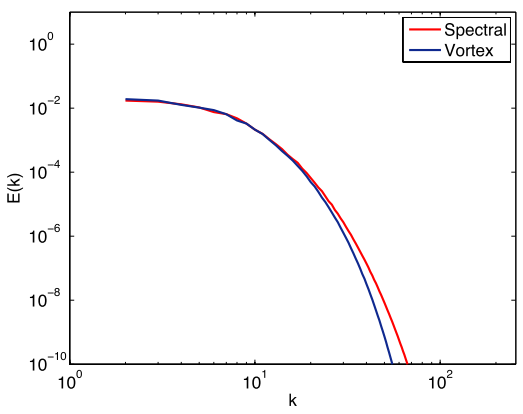

(b) Energy spectra at $t / T=10$

Fig. 3 Decay rate and spectrum of kinetic energy ${ }^{(31)}$

fourth order Runge-Kutta method for all terms. No forcing was applied to the calculation, since it would be difficult to do so with vortex methods. The spectral method was calculated on the same number of processors without using the GPUs.

The initial condition was generated in Fourier space as a solenoidal isotropic velocity field with random phases and a prescribed energy spectrum, and transformed to physical space. The spectral method calculation used this initial condition directly. The core radius of the vortex elements were set to $2 \pi / N^{1 / 3}$ so that the overlap ratio was 1 .

Figure 3(a) shows the decay of kinetic energy, which is defined as

$$
K=\frac{1}{2} \sum_{i=1}^{N} u_{i}^{2}+v_{i}^{2}+w_{i}^{2}
$$

"Spectral" is the pseudo-spectral method and "Vortex" is the vortex method calculation. The time is normalized by the eddy turnover time $T$. The integral scale and eddy turnover time have the following relation.

$$
\begin{aligned}
L & =\frac{\pi}{2 u^{\prime 2}} \int k^{-1} E(k) d k \\
T & =L / u^{\prime} .
\end{aligned}
$$

where $u^{\prime}=\frac{2}{3} K$. The homogeneous isotropic turbulence does not have any production of turbulence, and thus the kinetic energy decays monotonically with time. This decay rate is known to show a self-similar behavior at the finial period of decay. This is confirmed by the straight drop of $K$ that appears at the end of this log-log plot. The results of the two methods agree perfectly until $t / T=10$, where the kinetic energy drops an order of magnitude from the initial value.

Figure 3(b) shows the energy spectrum at $t / T=10 . k$ is the wave number, and $\mathrm{E}(\mathrm{k})$ is the kinetic energy contained in the wave number $k$. At this Reynolds number it is difficult to observe an inertial subrange of $k^{-5 / 3}$, nor a $k^{4}$ behavior at low wave numbers. The results of the two methods are in good agreement, except for the fact that the vortex method slightly underestimates the energy at higher wave numbers. This is a typical behavior of an under-resolved vortex method calculation. The inefficiency of discretizing the fields as a superposition of Gaussian distributions as opposed to a superposition of waves, requires the vortex method to use more points than the pseudo-spectral method.

The isosurface of the second invariant of the velocity derivative tensor $I I=u_{i, j} u_{j, i}$ at time $t / T=10$ is shown in Fig. 4. Fig. 4(a) is the isosurface of the spectral method, Fig. 4(b) shows the isosurface from the vortex method calculation. Although, the larger structures match between the two methods, the smaller structures behave differently. The difference in the small structures can also be observed in Fig. 3(b), where the kinetic energy at higher 


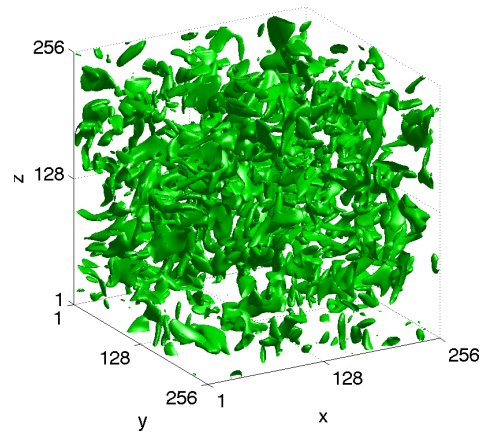

(a) Spectral method

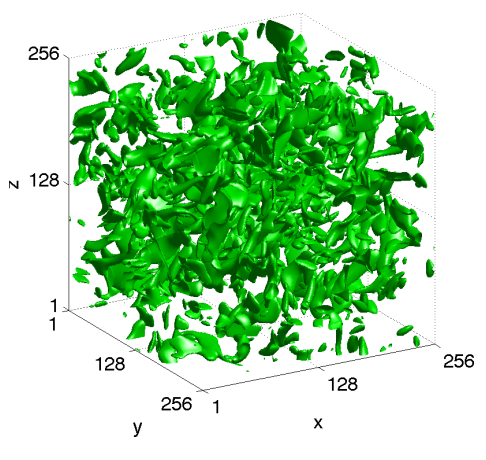

(b) Vortex method

Fig. 4 Isosurface of the second invariant (II) of the velocity derivative tensor ${ }^{(31)}$

wave numbers do not match. As mentioned in the previous paragraph, this is due to the discretization error of vortex methods, and is not an indication of the inconsistency of the method.

In summary, the isosurface of the second invariant, kinetic energy decay and energy spectrum of the well resolved vortex method calculation agreed quantitatively with that of the reference calculation using a spectral method.

\subsection{Homogeneous Shear Turbulence}

In this section, the vortex method calculation of the homogeneous shear flow is performed and compared with a finite difference method. The focus of this section is on the ability of vortex methods to reproduce the anisotropy of turbulence, i.e. the ability of spherical vortex blobs to reproduce streaky global vortex structures. The shear-rate is selected so that it matches the maximum shear-rate observed at $y^{+} \approx 10$ in near wall flows ${ }^{(49)}$. Another objective is the assessment of the production of turbulence in vortex methods, i.e. the validation of the vortex stretching term calculation for strongly strained flows. The present calculation of the homogeneous shear flow may be considered as an intermediate validation step, which will fill the gap between the calculation of isotropic turbulence and wall bounded flows.

Both the vortex method and finite difference method solve the Navier-Stokes equation in perturbation form, where the instantaneous velocity and vorticity are decomposed into the time-averaged component and the fluctuating component. The finite difference method is a SMAC method on a staggered grid. The fourth-order central difference method was used for the spatial discretization, and the third-order low storage Runge-Kutta method ${ }^{(51)}$ was used for the temporal discretization. The shear periodic boundary condition ${ }^{(50)}$ was used at the upper and lower boundaries. The vortex method uses the convergent core spreading method with reinitialization ${ }^{(31)}$. The shear-periodic boundary condition in the vortex method is calculated by an extension of the periodic FMM to handle shear ${ }^{(52)}$.

The homogeneous shear flow was calculated by the vortex method and the finite difference method, using $N=64^{3}, 128^{3}$ points. The initial Reynolds number based on Taylor's micro scale was $R e_{\lambda}=25$. The calculation domain was $[-\pi, \pi]^{3}$ for both methods. The initial condition of the homogeneous shear flow calculation (for both the vortex method and the finite difference method) were generated from the isotropic turbulence calculation described in the previous section.

One of the main objectives of the present study is to ascertain the magnitude of anisotropy, which the vortex blob method can handle. Lee et al. ${ }^{(49)}$ showed in their pseudo-spectral calculation of the homogeneous shear flow, that a high shear-rate produces streaky structures similar to those observed in the near-wall region of the channel flow DNS by Kim et al(53). 


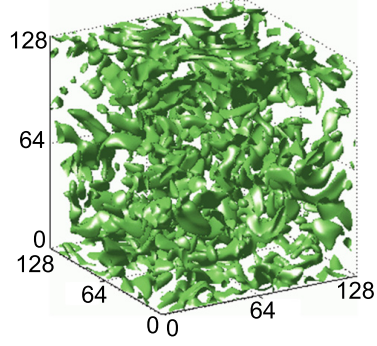

(a) $t^{*}=0$

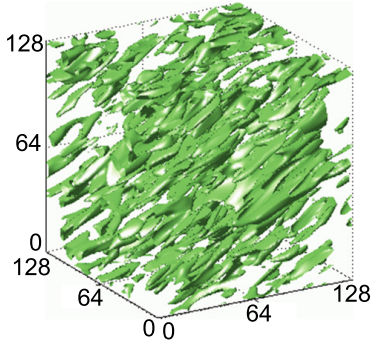

(b) $t^{*}=1$

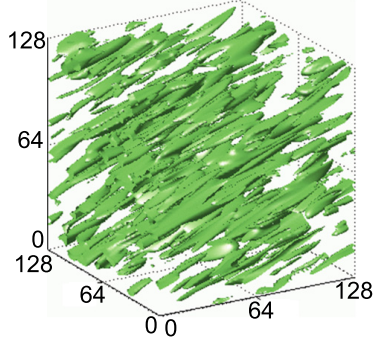

(c) $t^{*}=2$

Fig. 5 Isosurface of II (Finite Difference Method) ${ }^{(52)}$

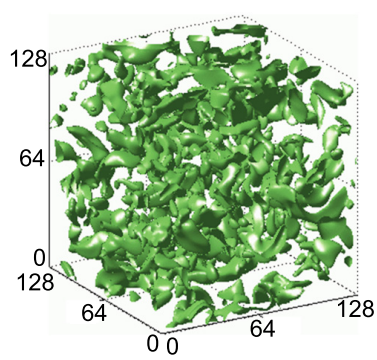

(a) $t^{*}=0$

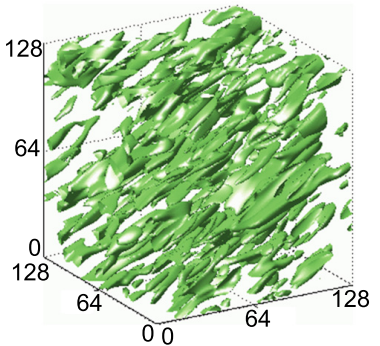

(b) $t^{*}=1$

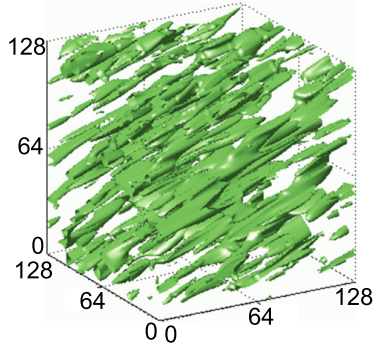

(c) $t^{*}=2$

Fig. 6 Isosurface of II (Vortex Method) ${ }^{(52)}$

They also investigated the suitable turbulence length scale that can be used to construct a dimensionless and universal shear-rate parameter, which can be used as an indicator of streaky structures for both the homogeneous shear flow and high shear regions near the wall. This dimensionless shear-rate parameter is defined as

$$
S^{*} \equiv \frac{2 S K}{\epsilon}
$$

where $K=\overline{u_{i}^{2}} / 2$ is the turbulent kinetic energy and $\epsilon=v \overline{u_{i, j} u_{j, i}}$ is the dissipation rate. Lee $e t$ $a l .{ }^{(49)}$ applied this shear-rate parameter to the fully developed channel flow by Kim et al. ${ }^{(53)}$ and showed that the maximum is $S^{*}=35$ in the viscous sublayer, and decreases to about one-sixth of the maximum in the logarithmic layer. In the present calculation the shear-rate $S=18$ is chosen so that the shear-rate parameter becomes $S^{*} \approx 35$.

The isosurface of the second invariant of the velocity gradient tensor $I I=u_{i, j} u_{j, i}$ for the finite difference method and vortex method are shown in Figs. 5 and 6, respectively. The time $t^{*}$ is normalized by the shear-rate $S$. At $t^{*}=0$, the flow is isotropic and the structures have no directional preference. At $t^{*}=1$, the vortices are strained and the structures begin to show a directional preference. At $t^{*}=2$, many streaky structures can be observed.

The Reynolds stress anisotropy tensor is a direct measure of the anisotropy of the velocity fluctuations, and is a more quantitative measure of the ability to reproduce anisotropic turbulence. The normal components of the Reynolds stress anisotropy tensor are shown in Fig. 7. $\operatorname{FDM}\left(64^{3}\right), \operatorname{VM}\left(64^{3}\right), \operatorname{FDM}\left(128^{3}\right)$, and $\operatorname{VM}\left(128^{3}\right)$ are the finite difference method using a $N=64^{3}$ grid, the vortex method using $N=64^{3}$ particles, the finite difference method using a $N=128^{3}$ grid, and the vortex method using $N=128^{3}$ particles. Different markers represent the different normal components of $b_{i j}$. The overall behavior of the present vortex method is similar to that of the finite difference method. The results of the two finite difference method calculations are indistinguishable. On the other hand, when the spatial resolution of 


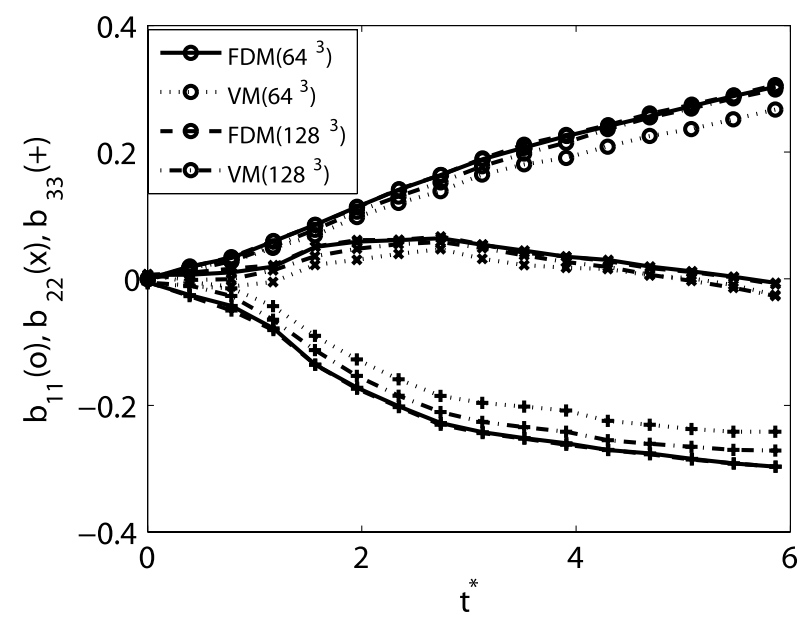

Fig. 7 The Development of the Reynolds Stress Anisotropy Tensor ${ }^{(52)}$

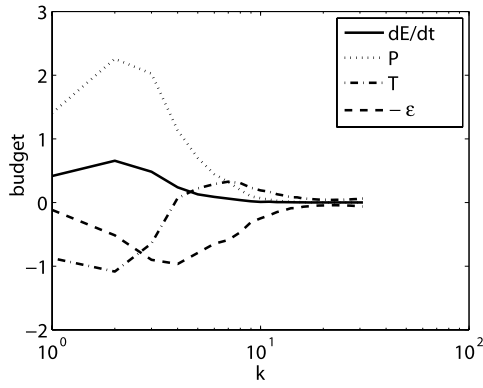

(a) FDM

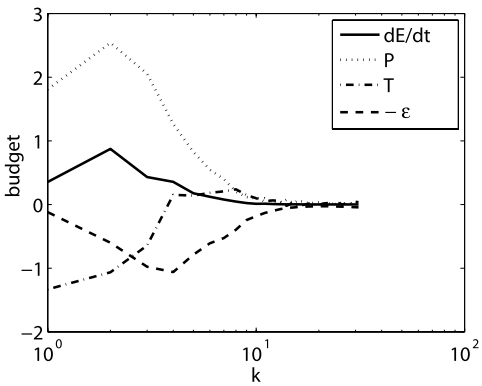

(b) VM

Fig. 8 Budget of the Energy Spectrum Equation at $t^{*}=2^{(52)}$

the vortex method is increased, the difference between the finite difference method and vortex method decreases. The fact that vortex methods require a larger number of elements compared to grid-based methods, is consistent with the observations in the isotropic turbulence calculations.

The remaining objective is the quantitative assessment of the ability of vortex methods to calculate the balance between production, transfer, and dissipation in the homogeneous shear flow. Since the production was not present in the isotropic turbulence, its quantitative assessment is of interest for the present homogeneous shear calculation.

The energy spectrum equation for the homogeneous shear flow can be written as

$$
\frac{\partial K}{\partial t}=\underbrace{S k_{1} \frac{\partial K}{\partial k_{3}}-S \mathcal{E}_{13}}_{\mathcal{P}}+\underbrace{\iota k_{k} \mathcal{T}_{i k, i}}_{\mathcal{T}}-\underbrace{2 v k^{2} K}_{\epsilon},
$$

where $\mathcal{E}_{i j}$ and $\mathcal{T}_{i j, k}$ are the two point double and triple velocity correlation tensors in wavenumber space, respectively. $K$ is the kinetic energy $K \equiv \frac{1}{2} \mathcal{E}_{i i}, S$ is the shear-rate, $k$ is the wavenumber, and $v$ is the kinematic viscosity. Each term on the right hand side of Eq. (16) is associated with an actual physical process. $\mathcal{P}$ represents the production, $\mathcal{T}$ represents the transfer, and $\epsilon$ represents the dissipation of the kinetic energy.

The budget of the above mentioned energy spectrum equation at $t^{*}=2$ for the finite difference method and vortex method is shown in Fig. 8. Although there are quantitative differences in the two plots, the overall tendency is very similar. First, the production has a 
peak at lower wavenumbers and vanishes at higher wavenumbers. This corresponds to the fact that the mean shear mainly influences the large structures of turbulence. Secondly, the transfer term has a large negative value at lower wavenumbers and has a positive value at higher wavenumbers. This reflects the fact that the energy drawn from the larger structures is gradually cascaded down to the smaller structures. Furthermore, the dissipation term has a negative peak at higher wavenumbers, although not too high for the present calculation at $R e_{\lambda}=25$. Finally, the change rate of kinetic energy shows an energy surplus, which is consistent with the fact that the total kinetic energy increases over time.

From these observations, it can be said that the present vortex method code can account for strong anisotropy comparable to that in the viscous sublayer of a near wall flow. Furthermore, it can quantitatively reproduce the balance between the production, transfer, and dissipation of kinetic energy if the spatial resolution is sufficient. Though, the number of necessary vortex elements may be large compared to grid-based methods.

\section{Engineering Applications}

In this section, we will introduce an example of the use of vortex methods in engineering applications of turbulent flows, e.g. the simulation of trailing edge vortices in aircraft wakes by Chatelain et al. ${ }^{(35)}$ Trailing edge vortices impose stringent safety requirements on distances between aircraft, limiting the landing and take-off capacities of airports. Several research efforts have focused on the identification of the governing physical mechanisms of wake evolution that would lead to the design of vortex wake alleviation schemes. Such schemes include the modification of the lift distribution to enhance sensitivity to instabilities ${ }^{(54)-(57)}$, and the introduction of suitable perturbations. ${ }^{(58)}$

In aircraft wakes, the vorticity is confined to a small region near the trailing edge vortices, and results in a large reduction of computational elements when discretized by vortex methods. Such flows are also dominated by unsteady vortex dynamics, which are most naturally solved by vortex methods. These features enabled the simulation of a full wake of an aircraft using a billion vortex particles on 16,000 BG/L processors. The authors use a parallel particle-mesh library $^{(27)}$ that uses domain decompositions and optimized data mappings to accelerate their vortex method calculation.

The vortex method calculation by Chatelain et al. ${ }^{(35)}$ was performed by simulating the onset of instabilities of multiple wavelengths in a long domain. Calculation conditions were similar to that of the LES calculation by Stumpf, ${ }^{(59)}$ except the Reynolds number was three times larger $\left(R e_{\Gamma_{1}}=6000\right)$. The domain length is chosen as the wavelength of maximum growth rate for the Crow instability, $L_{x}=9.4285 b_{1}$, where $b_{1}$ is the distance of the primary vortex pair. The transversal dimensions are $L_{y}=1 / 2 L_{x}$ and $L_{z}=3 / 8 L_{x}$. The vortices have Gaussian cores with $\sigma_{1} / b_{1}=0.05$ and $\sigma_{2} / b_{1}=0.025$. The secondary pair is located at $b_{2} / b_{1}=0.5$, with a relative strength $\Gamma_{2} / \Gamma_{1}=-0.35$, where $b_{2}$ is the distance of the secondary vortex pair. In addition to the initially unperturbed vortices, the vorticity field is filled with a white noise that produces $u_{R M S}=0.005 u_{\max }$.

Figure 9 shows the evolution of vorticity isosurfaces. Regions of high vorticity norm correspond to the red and opaque, while low vorticity are shown in blue and transparent. At $t / t_{0}=0.25$, we can see 10 and $11 \Omega$-loops along the two primary vortices. This corresponds to the average wavelengths $\lambda / b_{1}=0.943$ and 0.86 .

\section{Conclusions}

In the present review article, we have presented benchmark results for the vortex method for both speed and accuracy, along with some examples of its application to engineering turbulent flows. The benchmarks for speed were performed on different types of architectures, which offer vortex methods an advantage over traditional grid based methods. The runs on MDGRAPE-3 and GPUs have shown the potential of vortex methods as a highly parallel $O(N)$ algorithm. Since future computer architectures are becoming more and more parallel, we pre- 


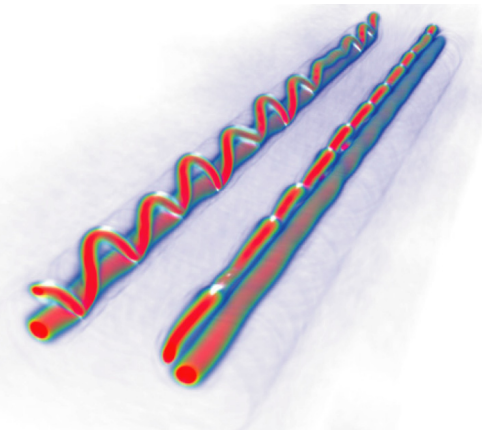

(a) $t / t_{0}=0.21$

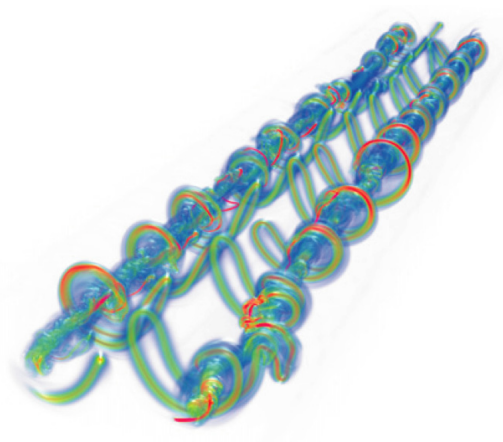

(c) $t / t_{0}=0.27$

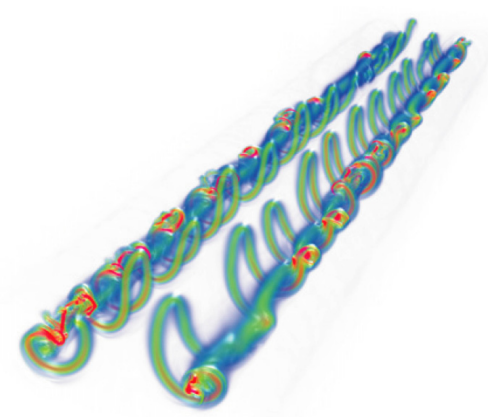

(b) $t / t_{0}=0.25$

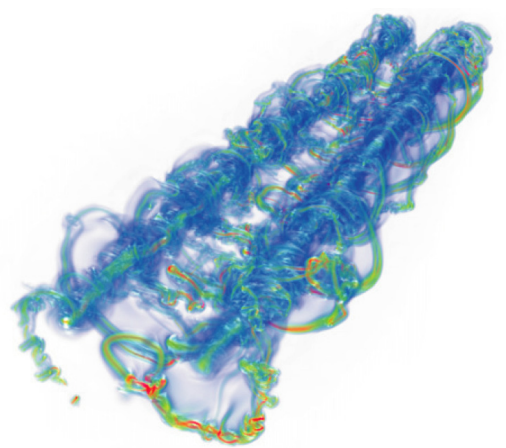

(d) $t / t_{0}=0.34$

Fig. 9 Counter-rotating vortices, initiation by ambient noise: visualization of the vorticity structures by volume rendering ${ }^{(35)}$. High vorticity norm regions correspond to red and opaque; low vorticity are blue and transparent. (a) $t / t_{0}=0.21$, (b) $t / t_{0}=0.25$, (c) $t / t_{0}=0.27$, and (d) $t / t_{0}=0.34$. 
dict that there will be an advantage of using FMMs, due to the high parallelism they offer. However, it is important to point out that there is a 100 fold difference in the calculation cost between FMMs and fast Poisson solvers at the moment, even on GPUs.

For the benchmarks for accuracy using the three canonical turbulent flows, the present vortex method has shown quantitative agreement with the reference calculations using finite difference and pseudo-spectral methods. The match of the decay rate of kinetic energy and the energy spectra in the homogeneous isotropic turbulence calculation exemplifies the high accuracy of the present viscous diffusion scheme. With this highly accurate viscous diffusion scheme, we are now able to capture the dissipation of kinetic energy correctly. By comparing vortex methods directly with spectral DNS, we are able to confirm that the solution of vortex methods converge to the Navier-Stokes solution. However, the number of vortex particles required seems to be much larger than spectral methods.

The fact that vortex methods were able to perform a direct numerical simulation ${ }^{(35)}$ of an aircraft wake of a Reynolds number that was three time larger than a state-of-the-art LES computation $^{(59)}$ demonstrates the possibility of vortex methods for the simulation of high Reynolds number turbulent flows. However, this is at the cost of using billions of vortex particles. As we have mentioned in the previous paragraph the number of vortex particles required to obtain an accurate solution is far larger than that of typical grid based methods. Cocle et al. estimates this number to be around 4-fold per dimension. ${ }^{(34)}$

There are numerous issues to be solved before the vortex method could become an efficient and reliable tool for the simulation of wall bounded turbulent flows. In order for the vortex method to become a reliable engineering tool the following areas must be further developed; anisotropic elements, high order basis functions, adaptive spatial refinement, hybrid fast-multipole particle-mesh methods, and their implementation on heterogeneous architectures.

\section{References}

( 1 ) Ould-Salihi, M. L. Cottet, \& G.-H. Hamraoui, M. E. (2000) Blending Finite-Difference and Vortex Methods for Incompressible Flow Computations. SIAM J. Sci. Comput. 22-5, 1655-1674.

( 2 ) Hamada, T., Yokota, R., Nitadori, K., Narumi, T., Yasuoka, K., Taiji, M., \& Oguri, K. (2009) 42 TFlops Hierarchical N-body Simulation on GPUs with Applications in both Astrophysics and Turbulence. in proc. SC'09, 1-12.

( 3 ) Rosenhead, L. (1931) The Formation of Vortices from a Surface of Discontinuity. Proc. Roy. Soc. London. Ser. A 134, 170-192.

( 4 ) Chorin, A. J. (1973) Discretization of a Vortex Sheet, with an Example of Roll-Up. J. Comput. Phys. 13-3, 423-429.

( 5 ) Kuwahara, K. \& Takami, H. (1973) Numerical Studies of Two-Dimensional Vortex Motion by a System of Point Vortices. J. Phys. Soc. Japan 34-1, 247-253.

( 6 ) Hald, O. H. (1978) Convergnece of Vortex Methods for Euler's Equations. Math. Comput. 32-143, 791-809.

( 7 ) Beale, J. T. \& Majda, A. (1982) Vortex Methods. I: Convergence in Three Dimensions. Math. Comput. 39-159, 1-27.

( 8 ) Hess, J. L. (1990) Panel Methods in Computational Fluid Dynamics. Annu. Rev. Fluid Mech. 22, 255-274.

( 9 ) Pozrikidis, C. (2002) A Practical Guide to Boundary Element Methods., Chapman \& Hall/CRC.

(10) Cottet, G.-H. \& Koumoutsakos, P. D. (2000) Vortex Methods, Cambridge University Press.

(11) Chorin, A. J. (1973) Numerical Study of Slightly Viscous Flow. J. Fluid Mech. 57, 785796. 
(12) Leonard, A. (1980) Vortex Methods for Flow Simulations. J. Comput. Phys. 37, 289335.

(13) Degond, P. \& Mas-Gallic, S. (1989) The Weighted Particle Method for ConvectionDiffusion Equations. Part 1: The Case of an Isotropic Viscosity. Math. Comput. 53-188, 485-507.

(14) Couet, B. \& Buneman, O. (1981) Simulation of Three-Dimensional Incompressible Flows with a Vortex-In-Cell Method. J. Comput. Phys. 39-2, 305-328.

(15) Chorin, A. J. (1978) Vortex Sheet Approximation of Boundary Layers. J. Comput. Phys. 27-3, 428-442.

(16) Fishelov, D. (1990) Vortex Methods for Slightly Viscous Three-Dimensional Flow. SIAM J. Sci. Stat. Comput. 11-3, 399-424.

(17) Winckelmans, G. S. \& Leonard, A. (1993) Contributions to Vortex Particle Methods for the Computation of Three-Dimensional Incompressible Unsteady Flows. J. Comput. Phys. 109, 247-273.

(18) Cottet, G.-H., Koumoutsakos, P., \& Ould Salihi, M. L. (2000) Vortex Methods with Spatially Varying Cores. J. Comput. Phys. 162-1, 164-185.

(19) Daeninck, G., Ploumhans, P., \& Winckelmans, G. S. (2002) Simulation of ThreeDimensional Bluff-Body Flows Using Vortex Methods: From Direct Numerical Simulation Towards Large-Eddy Simulation Modeling. J. Turb. 043, 1-7.

(20) Koumoutsakos, P., Leonard, A., \& Pepin, F. (1994) Boundary Conditions for Viscous Vortex Methods. J. Comput. Phys. 113-1, 52-61.

(21) Ploumhans, P. \& Winckelmans, G. S. (2000) Vortex Methods for High-Resolution Simulations of Viscous Flow Past Bluff Bodies of General Geometry. J. Comput. Phys. 165-2, 354-406.

(22) Ploumhans, P., Winckelmans, G. S., Salmon, J. K., Leonard, A., \& Warren, M. S. (2002) Vortex Methods for Direct Numerical Simulation of Three-Dimensional Bluff Body Flows: Application to the Sphere at $\mathrm{Re}=300,500$, and 1000. J. Comput. Phys. 178-2, 427-463.

(23) Barnes, J. \& Hut, P. (1986) A Hierarchical $O(N \log N)$ Force-Calculation Algorithm. Nature 324, 446-449.

(24) Greengard, L. \& Rokhlin, V. (1987) A Fast Algorithm for Particle Simulations. J. Comput. Phys. 73, 325-348.

(25) Sugimoto, D., Chikada, Y., Makino, J., Ito, T., Ebisuzaki, T., Umemura, M. (1990) A Special-purpose Computer for Gravitational Many-body Problems. Nature 345, 33-35.

(26) Fukushige, T., Taiji, M., Makino, J., Ebisuzaki, T., \& Sugimoto, D. (1996) Parallelized Special-purpose Computer for Many-body Simulations with an Arbitrary Central Force. Astrophys. J. 468, 51-61.

(27) Sbalzarini, I. F., Walther, J. H., Bergdorf, M., Hieber, S. E., Kotsalis, E. M., \& Koumoutsakos, P. (2006) PPM-A Highly Efficient Parallel Particle-Mesh Library for the Simulation of Continuum Systems. J. Comput. Phys. 215, 566-588.

(28) Poncet, P. (2006) Finite Difference Stencils Based on Particle Strength Exchange Schemes for Improvement of Vortex Methods. J. Turb. 7-23, 1-24.

(29) Rossi, L. F. (2005) Achieving High-Order Convergence Rates with Deforming Basis Functions. SIAM J. Sci. Comput. 26-3, 885-906.

(30) Ploumhans, P., Daeninck, G., \& Winckelmans, G. (2004) Simulation of ThreeDimensional Bluff-Body Flows Using the Vortex Particle and Boundary Element Methods. Flow Turb. Combust. 73-2, 117-131.

(31) Yokota, R., Sheel, T. K., \& Obi, S. (2007) Calculation of Isotropic Turbulence Using a Pure Lagrangian Vortex Method J. Comput. Phys. 226-2, 1589-1606.

(32) Winckelmans, G. S., Salmon, J. K., Warren, M. S., Leonard, A., Jodoin, B. (1996) Application of Fast Parallel and Sequential Tree Codes to Computing Three-Dimensional Flows with the Vortex Element and Boundary Element Methods. ESAIM Proc. 1, 225- 
240.

(33) Marzouk, Y. M. \& Ghoniem A. F. (2005) K-means Clustering for Optimal Partitioning and Dynamic Load Balancing of Parallel Hierarchical N-Body Simulations J. Comput. Phys. 207, 493-528.

(34) Cocle, R., Winckelmans, G., Daeninck, G. (2008) Combining the Vortex-In-Cell and Parallel Fast Multipole Methods for Efficient Domain Decomposition Simulations. $J$. Comput. Phys. 227, 2263-2292.

(35) Chatelain, P., Curioni, A., Bergdorf, M., Rossinelli, D., Anderoni, W., \& Koumoutsakos, P. (2008) Billion Vortex Particle Direct Numerical Simulation of Aircraft Wakes. Comput. Methods Appl. Mech. Engrg. 197, 1296-1304.

(36) Sheel, T. K., Yasuoka, K., \& Obi, S. (2007) Fast vortex method calculation using a special-purpose computer. Comput. Fluids 36-8, 1319-1326.

(37) Sheel, T. K., Yokota, R., Yasuoka, K., \& Obi, S. (2008) The study of colliding vortex rings using a special-purpose computer and FMM Trans. Jap. Soc.Comput. Eng. Sci., 20080003.

(38) Nyland, L., Harris, M., \& Prins, J. (2007) Fast N-body Simulation with CUDA. GPU Gems 3, ed. H. Nguyen, 677-695.

(39) Belleman, R. G., Bedorf, J., \& Portegies Zwart,S. F. (2008) High Performance Direct Gravitational N-body Simulations on Graphics Processing Units ii: An Implementation in CUDA. New Astronomy 13, 103-112.

(40) Hamada, T. \& Iitaka, T. (2007) The Chamomile Scheme: An Optimized Algorithm for N-body Simulations on Programmable Graphics Processing Units. ArXiv Astrophysics e-prints astro-ph/073100.

(41) Schive, H.-Y., Chien, C.-H., Wong, S.-K., Tsai, Y.-C., \& Chiueh, T. (2007) Graphic-Card Cluster for Astrophysics (GraCCA) -Performance Tests. ArXiv Astrophysics e-prints astro-ph:0707.2991v1.

(42) Stone, J. E., Phillips, J. C., Freddolino, P. L., Hardy, D. J., Trabuco, L. G., \& Schulten, K. (2007) Accelerating Molecular Modeling Applications with Graphics Processors. J. Comput. Chem. 28, 2618-2640.

(43) Stock, M. J. \& Gharakhani, A. (2008) Toward Efficient GPU-accelerated N-body Simulations. AIAA paper, 2008-608, 1-13.

(44) Gumerov, N. A. \& Duraiswami, R. (2008) Fast Multipole Methods on Graphics Processors. J. Comput. Phys. 227 8290-8313.

(45) Yokota, R., Narumi, T., Sakamaki, R., Kameoka, S., Obi, S. \& Yasuoka, K. (2009) Fast Multipole Methods on a Cluster of GPUs for the Meshless Simulation of Turbulence. Comput. Phys. Comm. 11, 2066-2078.

(46) Cottet, G. H., Michaux, B., Ossia, S., \& Van der Linden, G. (2002) A Comparison of Spectral and Vortex Methods in Three-Dimensional Incompressible Flows. J. Comput. Phys 175, 702-712.

(47) Totsuka, Y. \& Obi, S. (2007) A Validation of the Viscous Dissipation Model for Fast Vortex Methods in Simulations of Decaying Turbulence. J. Fluid Sci. Tech. 2, 248-257.

(48) Rogallo, R. S. (1981) Numerical experiments in homogeneous turbulence. NASA Tech. Mem., 81315.

(49) Lee, M. J., Kim, J., \& Moin, P. (1990) Structure of Turbulence at High Shear Rate. J. Fluid Mech. 216, 561-583.

(50) Schumann, U. (1985) Algorithms for Direct Numerical Simulation of Shear-Periodic Turbulence, in: Soubbaramayer. Proc. 9th Int. Conf. Num. Meth. Fluid Dyn., Lecture Notes in Physics 218.

(51) Williamson, J. H. (1980) Low-storage Runge-Kutta Schemes. J. Comput. Phys. 35, 4856.

(52) Yokota, R. \& Obi, S. (2010) Comparing Vortex Methods and Finite Difference Methods in a Homogeneous Shear Flow. Int. J. Num. Meth. Fluids, 63-7, 828-846. 
(53) Kim, J., Moin, P., \& Moser, R. D. (1987) Turbulence Statistics in Fully Developed Channel Flow at Low Reynolds Number. J. Fluid Mech. 177, 133-166.

(54) Crouch, J.D. (1997) Instability and transient growth for two trailing-vortex pairs. J. Fluid Mech. 350, 311-330.

(55) Durston, D.A., Walker, S.M., Driver, D.M., Smith, S.C., Savas, O. (2005) Wake vortex alleviation flow field studies. J. Aircraft 42-4, 894-907.

(56) Graham, W.R., Park, S.-W., Nickels, T.B. (2003) Trailing vortices from a wing with a notched lift distribution. AIAA J. 41-9, 1835-1838.

(57) Ortega, J.M., Savas, O. (2001) Rapidly growing instability mode in trailing multiplevortex wakes. AIAA J. 39-4, 750-754.

(58) Crouch, J.D., Miller, G.D., Spalart, P.R. (2001) Active-control system for breakup of airplane trailing vortices. AIAA J. 39-12, 2374-2381.

(59) Stumpf, E. (2005) Study of four-vortex aircraft wakes and layout of corresponding aircraft configurations. J. Aircraft 42-3, 722-730. 